\title{
Unidades de aprendizaje, una propuesta de complemento a los objetos de aprendizaje.
}

\author{
Eduardo Hernández \\ eduardoestatusquo.cl \\ Consultor y Asesor Independiente en elearninng. \\ Ingeniero en Computación e Informática
}

\section{Introducción}

Tal cual como se ha venido desarrollando el concepto de Learning Object (LO) hasta hoy, éste abarca principalmente a un conjunto de materiales digitales los que como unidad o agrupación permiten o facilitan alcanzar un objetivo educacional. Las actuales iniciativas de especificación y estandarización del eLearning, como SCORM (Sharable Content Object Reference Model) e IMS, se han preocupado en hacer de estos LO, recursos que puedan ser interoperables, es decir que funcionen y sean comprendidos por distintas soluciones computacionales.

La práctica y las distintas definiciones existentes actualmente ligan, expresamente o tácitamente, el concepto Learning Object con la idea de "contenidos" o "recursos digitales".

Por ejemplo, Wiley escribe "...Por lo tanto, este capítulo definirá Learning Objects como "cualquier recurso digital que puede ser reutilizado para facilitar el aprendizaje". Esta definición incluye a cualquier cosa que pueda ser distribuida a través de una red bajo demanda, sea grande o pequeña. Ejemplos de los recursos digitales reutilizables más pequeños incluyen a imágenes o fotos, datos en vivo, cortos de video o audio pregrabados o en vivo, pequeñas porciones de texto, animaciones, pequeñas aplicaciones Web, como una calculadora hecha en Java. Ejemplos de recursos digitales reutilizables de mayor tamaño son páginas Web completas que combinen texto, imágenes y otros medios de comunicación..."(Wiley, 2000)

El párrafo anterior no hace sino confirmar el énfasis que se hace a la asociación del concepto Learning Object con contenidos, y sin ir más lejos, las aplicaciones, conocidas como "Repositorios" que alojan y permiten recuperar los Learning Objects, son, en la práctica, verdaderas bóvedas de contenidos esperando a ser reutilizados.

Las actuales tecnologías, como los estándares y especificaciones del eLearning (SCORM, IMS, IEEE), así como el nacimiento de estos repositorios han permitido la clasificación, almacenaje, búsqueda y recuperación de LOs promoviendo así los conceptos de reutilización e interoperabilidad.

Un análisis de la funcionalidad de dichos estándares, como SCORM, revelan que el proceso por el cual un alumno aprende es atravesando una serie de Learning Objects y en donde, además, dicho "viaje" es realizado de manera individual y totalmente independiente. Desde este punto de vista, se puede concluir que las especificaciones actuales potencian o promueven el sentimiento que el aprendizaje es el consumo de Learning Objects.

Por otro lado, las actuales tecnologías de interoperabilidad hacen que la enseñanza sea vista como la "ciencia de encontrar, seleccionar y ordenar los Learning Objects adecuados" (Koper y Olivier, 2004). Los autores establecen que usando la pedagogía promovida por los actuales estándares, la enseñanza es vista como el arte de seleccionar y ofrecer contenido en una manera secuenciada y estructurada, para luego registrar el progreso del alumno y, finalmente, evaluar el conocimiento adquirido. 
Con las especificaciones actuales existe un sentimiento de incertidumbre, un sentimiento que los Learning Objects están llevando a ver el eLearning como un secuencia de páginas que seguir, y a un "contenido estático, fosilizado, y muerto", con una baja motivación y compromiso por parte del alumno, y en ambientes impersonales y aislados", centrándose principalmente en un eLearning donde un alumno en solitario se sienta frente a la computadora, con un mecanismo encargado de realizar un seguimiento del rendimiento de una manera totalmente individual alumno por alumno. (Tattersal et al., 2003)

En la práctica docente actual, apoyada por tecnologías de la información y comunicación, podemos encontrar, efectivamente, el uso de contenidos (Learning Objects) pero éstos son utilizados y acompañados, en muchas ocasiones, de un conjunto de otros servicios como el correo electrónico, foros, Chat, etc. Dicho de una manera coloquial y fácil de comprender: generalmente un profesor o tutor hace uso de "Learning Objects y los potencia con algo más" para alcanzar su objetivo, en donde ese "algo más" son actividades o situaciones impartidas ya sea a distancia o presenciales que forman parte del modelo pedagógico seguido por el profesor o tutor. Incluso debemos ser capaces de reconocer que el aprendizaje podría producirse sin la presencia de Learning Objects.

Todo lo anteriormente descrito nos hace concluir que un curso o una lección claramente no consisten, muchas veces, sólo de contenidos, por lo tanto lograr la descripción, diseño e interoperabilidad completa de un curso es algo más que las actuales especificaciones. Un curso, una lección constituye algo más complejo que puede involucrar la participación de varias personas (alumnos, tutores) las que desarrollan una serie de actividades bajo un método o modelo pedagógico que puede ser, por ejemplo, un ambiente de trabajo personal o un ambiente de trabajo comunitario multiusuario.

Las actuales prácticas pedagógicas no consideran al alumno como una persona aislada, y en la mayoría de los casos se ven involucrados otros roles que interactúan entre sí. Tutores y mentores se relacionan con alumnos, y los alumnos interactúan con ellos. Los más nuevos modelos como la pedagogía constructivista, el aprendizaje basado en problemas poseen características en donde el consumo de contenidos no es precisamente lo que se busca o desea.

Uno de los principios educativos asociados con una concepción constructivista del aprendizaje es que, justamente, el aprendizaje se facilita gracias a la mediación o interacción con los otros, por lo tanto, es social y cooperativo. (Díaz-Barriga y Hernández, 2002)

De esta manera, se puede concluir que las especificaciones y estándares actuales de eLearning, basadas completamente en los LOs, deben ser extendidas para permitir incluir estas complejas interacciones y roles, así como los variados modelos pedagógicos existentes.

Una especificación que permita describir los modelos pedagógicos actuales y que además sea interoperable debe cumplir, principalmente, con las siguientes características (Koper y Olivier, 2004):

1) Completa: Una especificación debe permitir describir de manera completa un proceso de enseñanza y aprendizaje, sin imponer tácita o expresamente una en especial. Dentro de los elementos que debe permitir se encuentran:

a. Descripción y asignación de roles de las personas involucradas (alumnos, profesores, tutores, etc.)

b. Descripción e integración de las actividades desarrolladas por el alumno y el cuerpo docente.

c. Descripción e integración de los recursos que se usarán al interior del proceso de aprendizaje, siendo los Learning Objects uno de los posibles recursos y totalmente optativos.

d. Soporte para un modelo de aprendizaje individual y grupal. 
e. Soporte para modelos que no se basen puramente en un ambiente en línea, como el blended learning, por ejemplo.

2) Pedagógicamente expresivo: La especificación debe además brindar la posibilidad de poder expresar el significado pedagógico de los elementos involucrados.

3) Personalización: La especificación debe permitir que los recursos y actividades involucradas en un proceso de aprendizaje se adapten a las preferencias, necesidades, conocimiento previo o circunstancias de los involucrados, sean éstos alumnos, personal docente, o incluso la misma computadora.

4) Compatible: La especificación que permita diseñar aprendizaje más complejos deberá integrarse con las especificaciones ya existentes, como IMS, SCORM, IEEE.

5) Reutilizable e interoperable: Una característica compartida y ya lograda por las actuales especificaciones que permita, a través de una tecnología estándar, volver a utilizar recursos ya diseñados y creados una vez.

\section{Una posible solución}

El consorcio IMS ha diseñado y publicado una nueva especificación conocida como IMS Learning Design (IMS LD) que pretende subsanar y encontrar una posible solución a esta actual situación.

"Learning Design" que se traduce al español como "Diseño del Aprendizaje", se deriva de otra expresión muy escuchada y conocida "Instructional Design" (Diseño Instruccional). El uso de la palabra learning (aprendizaje) ayuda a enfatizar la variedad de métodos y mecanismos que van más allá de "enseñar o impartir conocimiento" que está más asociado con el diseño instruccional.

Un Diseño de Aprendizaje (Learning Design) es definido en la especificación IMS como "una descripción de un método que permite a los alumnos alcanzar ciertos objetivos de aprendizaje por medio del desarrollo de ciertas actividades de aprendizaje en un cierto orden en el contexto de un cierto ambiente de aprendizaje" (IMS, 2003).

También se le define como la aplicación de un modelo pedagógico para un objetivo de aprendizaje, un grupo objetivo y un contexto determinado (Koper y Olivier, 2004). En realidad, IMS Learning Design permite que a través de una especificación aceptada públicamente se exprese un proceso de enseñanzaaprendizaje, lo que equivale a describir las actividades y los recursos de ellas, que serán utilizadas y desarrolladas por personas (alumnos y tutores) para permitir que los alumnos alcancen un objetivo educacional esperado.

Esta especificación de IMS plantea que el Diseño de Aprendizaje, es decir la descripción del proceso enseñanza-aprendizaje unido a los recursos físicos que dicho proceso hará uso, incluyendo Learning Objects, se puede "empaquetar" en una única entidad llamada Unit of Learning también conocida como UOL, que traducida a nuestro idioma sería llamada "Unidad de Aprendizaje".

\section{Estructura de IMS Learning Design}

La nueva especificación de IMS presenta una serie de elementos medulares, que se presentan en la Imagen 1. (IMS, 2003) 


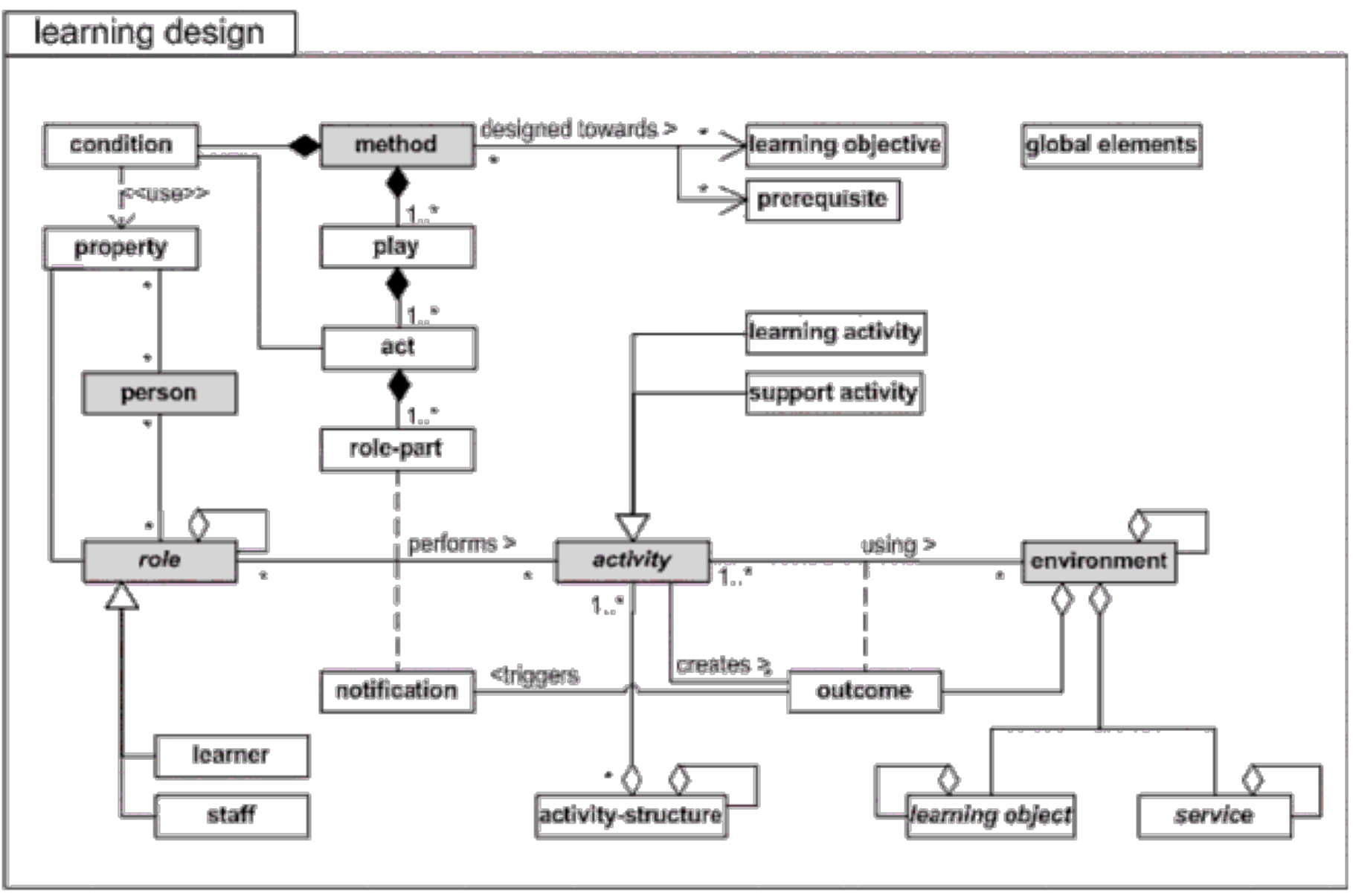

\section{Imagen 1 - Estructura General de IMS LD}

Según esta especificación un aprendizaje se diseña representando la siguiente idea: una persona (person) adquiere un rol (role) en el proceso de enseñanza-aprendizaje, usualmente este rol será de alumno (learner) o miembro del equipo (staff) docente, como un tutor, mentor. Dentro del rol, las personas desarrollarán actividades (activities) para lograr ciertas salidas o productos (outcomes). Las actividades bajo esta especificación podrán ser de aprendizaje (learning activities) o de soporte (support activities). Las actividades son desarrolladas dentro de un ambiente (environment), el que consiste de los servicios (services) y los objetos de aprendizaje (learning objects) necesarios o apropiados para que los roles desarrollen las actividades. Así, entonces Le arning Objects creados bajo la especificación SCORM o IMS podrían ser parte de un Learning Design.

El método (method) es el mecanismo que provee la especificación para coordinar los roles, las actividades y los ambientes de manera de permitir a los alumnos alcanzar los objetivos de aprendizaje.

Los elementos descritos anteriormente constituyen los elementos de base de IMS LD y se les conoce como Level A (Nivel A).

Luego, la especificación posee el Nivel B, el cual añade Propiedades y Condiciones (conditions) al Nivel A, lo que permite la personalización así como secuencias e interacciones más elaboradas basadas, por ejemplo, en los portafolios de los alumnos. Las propiedades pueden ser utilizadas para dirigir las actividades de aprendizaje así también como para registrar los resultados.

El último nivel conocido como Nivel C añade notificaciones (notifications) al Nivel B. Una notificación se gatilla u origina por un resultado de una actividad y puede producir que una nueva actividad quede disponible para el rol que se está ejecutando.

Cada uno de los niveles es representado a través del lenguaje XML (eXtensible Markup Language), con lo cual se logra alcanzar el objetivo anteriormente descrito que buscaba encontrar la compatibilidad entre 
esta nueva especificación y las ya existentes, también basadas en XML.

Según Tattersal, en la ausencia de una manera estandarizada de describir los procesos de aprendizaje, los diseñadores hoy utilizan HTML (HyperText Markup Language), lenguajes, o herramientas propietarias para escribir las secuencias de actividades a ser desarrolladas por los alumnos, para establecer la comunicación, para almacenar los resultados de las interacciones, y la mayoría de los eventos al interior de un proceso de enseñanza-aprendizaje.

Un diseño de aprendizaje descrito con IMS LD se traduce a un archivo XML estandarizado que incluye referencias a todos los elementos (roles, actividades, ambientes, servicios, recursos). La generación de este archivo puede hacerse utilizando aplicaciones de software las que a través de una interfaz gráfica ocultan la complejidad del lenguaje XML. A estas aplicaciones se las ha llamado "Editoras de IMS Learning Design" o "Editoras de Diseño de Aprendizaje".

Para facilitar su desarrollo e implementación una Unidad de Aprendizaje toma la idea de un paquete de contenidos de IMS Content Packaging (IMS, 2003). Ver Imagen 2 e Imagen 3.

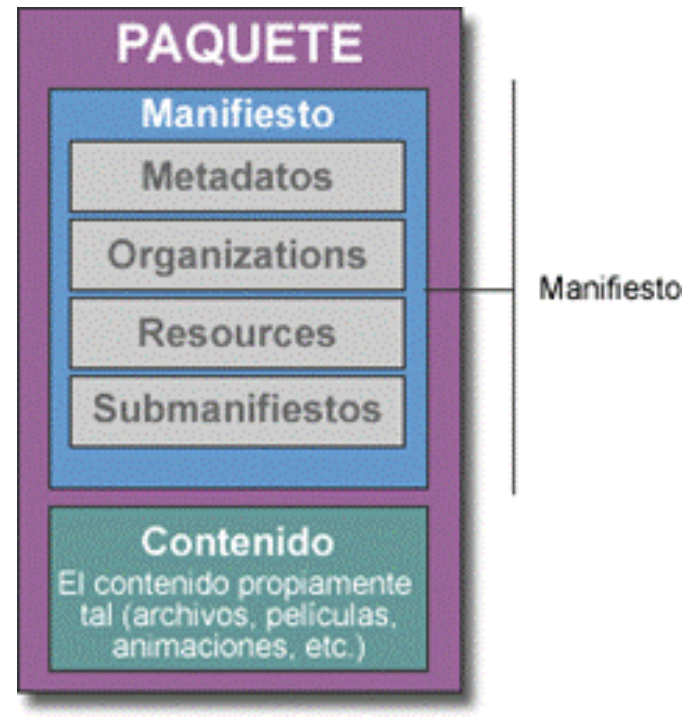

Imagen 2 - Estructura de un paquete IMS Content Packaging

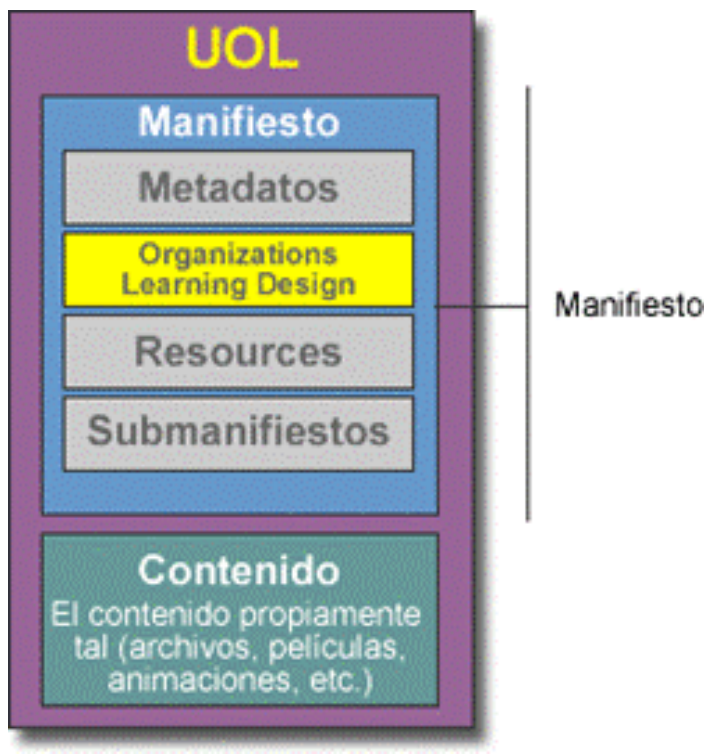

Imagen 3 - Estructura de una Unidad de Aprendizaje (UOL) en IMS Learning Design

Posteriormente, dicho archivo XML debe ser cargado y reproducido por un "Reproductor de Diseños de Aprendizaje" (Learning Design Player) que permita asignar usuarios o personas a los distintos roles para que éstos ejecuten, a través del mismo Reproductor, las actividades dentro de un ambiente. El reproductor 
puede ser una aplicación independiente o bien podría ser un sistema ya existente, al cual se le crea o añade una extensión para soportar IMS Learning Design.

\section{Situación actual de implementación}

En la actualidad existen distintos esfuerzos de llevar a la práctica la especificación IMS LD que se pasan a revisar.

RELOAD Learning Design Editor, (http://www.reload.ac.uk/) es una aplicación, de código abierto, que permite la generación de learning objects y paquetes bajo la especificación IMS y SCORM, además de permitir la creación y edición de metadatos. Ahora ha añadido soporte para IMS LD Nivel A, B y C.

CopperCore, (http://coppercore.org/) es un reproductor IMS LD de código abierto capaz de leer e interpretar Unidades de Aprendizaje bajo IMS LD, asignndo personas al diseño de aprendizaje de manera que ellas ejecuten los roles establecidos en el diseño. CopperCore implementa IMS LD en sus tres niveles.

RELOAD Learning Design Player, (http://www .reload.ac.uk/) es un reproductor de IMS LD, basado en CooperCore, y desarrollado en la Universidad de Bolton por Paul Sharples y Phillip Beauvoir. Construido y distribuido como aplicación de código abierto.

eLiveSuite (http://www.elive-ld.com/) es un editor, de origen Alemán, que permite trabajar con los Niveles A y B de la especificación.

ASK Learning Design Toolkit (http://www.ask.iti.gr/index.html) consiste de una aplicación de origen Griego que permite trabajar con IMS LD soportando hasta el Nivel B.

LAMS -Learning Activity Management System- (http://www.lamsinternational.com/) es una aplicación que permite realizar todas la fases involucradas en IMS LD; es decir, permite la generación de diseño de aprendizaje a través de una interfaz de autor, para luego permitir la asignación de personas a los distintos roles del diseño, y finalmente permite que los usuarios se conecten al sistema para permitir la ejecución de sus actividades y completar el diseño de aprendizaje. Si es importante destacar que LAMS nace como una implementación IMS LD pero que durante su fase de desarrollo debió apartarse ligeramente de la especificación al encontrar ciertas complicaciones en su implementación. Así, entonces los creadores del sistema han optado por establecer que LAMS está basado o inspirado en IMS LD.

\section{Soluciones ofrecidas por IMS}

Sin mecanismos acordados y compatibles que describan las estrategias de enseñanza, los creadores de materiales de enseñanza y la organización de éstos continúan experimentando dificultades innecesarias en:

- Documentar las estrategias utilizadas al interior de estos materiales.

- Apego a procedimientos preestablecidos para asegurar la consistencia de la documentación.

- Asegurar que la calidad de la enseñanza se cumple a través y entre organizaciones.

- Reutilización de elementos de materiales existentes de enseñanza.

- Reutilización e interoperabilidad no sólo de los recursos sino de un diseño de aprendizaje.

IMS LD provee un nivel de abstracción en el proceso, ofreciendo piezas genéricas para diferentes enfoques pedagógicos. Utilizando el lenguaje, los diseñadores pueden conversar en términos más de pedagogía que de tecnología, haciendo, por lo tanto, explícitas las alternativas pedagógicas sujetas a revisión, inspección, crítica y comparación.

\section{Conclusiones}

El mecanismo de IMS LD propuesto en este trabajo para el diseño y creación de procesos enseñanza- 
aprendizaje de mayor complejidad permite modelar distintos sistemas pedagógicos. Las pruebas con la especificación y documentadas por Koper y Olivier reportan que la especificación ha sido exitosa en diseñar aprendizaje centrado en el alumno, aprendizaje centrado en el conocimiento, centrado en la evaluación y centrado en comunidades, así también como ha demostrado efectivamente modelar aprendizajes basados en problemas o basado en actividades (Aprender haciendo).

Se espera que con el tiempo, la aparición y la madurez de editores y reproductores la comunidad educativa comience a trabajar con la especificación para comprobar la efectividad de ella en el modelamiento de procesos de enseñanza y aprendizaje. De esta manera, IMS LD puede transformarse no sólo en una herramienta no sólo para lograr la ejecución e interoperabilidad de diseños de aprendizaje sino también se puede convertir en un mecanismo de especificación del diseño instruccional al interior de una institución, unificando así la metodología para construir modelos pedagógicos al interior de una institución.

Sin embargo y a pesar de estos éxitos, la especificación hoy carece de una implementación de software que efectivamente permita validar la interoperabilidad del Diseño de Aprendizaje. Además, se debe tomar en cuenta la experiencia que han tenido terceros, como el sistema LAMS en donde se encontró problemas en la implementación de IMS LD, lo cual podría llevar a una revisión de la actual especificación corrigiendo ciertos errores o ambigüedades.

IMS LD se plantea como una capa superior a los actuales Learning Objects, en donde éstos pasan a ser elementos que podrían verse involucrados en un Diseño de Aprendizaje. Así, entonces se concluye que IMS Learning Design no reformula, altera o cambia el significado de los Objetos de Aprendizaje y al utilizar la misma tecnología (XML) y conceptos (la Unidad de Aprendizaje es un paquete) su integración con las actuales especificaciones es totalmente posible, como SCORM, IMS QTI (Question and Test Interoperability), por ejemplo.

La posibilidad de incorporar Learning Objects SCORM y recursos IMS QTI al interior de un IMS Learning Design plantea el desafío que los reproductores IMS LD no sólo deberán ser capaces de interpretar el Learning Design sino también de proveer los mecanismos para hacer funcionales a los Learning Objects que sigan la especificación SCORM o motores de interpretación de IMS QTI.

\section{Referencias Bibliográficas:}

DÍAZ-BARRIGA, F. y HERNÁNDEZ, G. (2002). Estrategia Docentes para un aprendizaje significativo ( $2^{a}$ Edición). Ciudad de México, Editorial McGraw-Hill Interamericana

IMS GLOBAL LEARNING CONSORTIUM, INC. (2003), IMS Learning Design Information Model 1.0. Recuperado desde http://www.imsglobal.org

IMS GLOBAL LEARNING CONSORTIUM, INC. (2003), IMS Learning Design Best Practice and Implementation Guide Recuperado desde http://www.imsglobal.org

KOPER, R., \& OLIVIER, B. (2004). Representing the Learning Design of Units of Learning. Educational Technology \& Society,7 (3), 97-111.

TATTERSAL et al. (2003), IMS Learning Design Frequently Asked Question. Recuperado desde http://learningnetworks.org/downloads/IMSLD/IMS\%20Learning\%20Design\%20FAQ\%201.0.pdf

WILY, D. (2002). "Connecting learning objects to instructional design theory: A definition, a metaphor, and a taxonomy", AIT/AECT, The Instructional Use of Learning Objects, Association for Instructional Technology, 1-35 
(C) Ediciones Universidad de Salamanca 